\section{What is an Olfactory Hallucination?}

\section{Robert I Henkin*}

The Taste and Smell Clinic, Washington D.C., USA

\section{Editorial}

An olfactory hallucination is the perception of an abnormal (distorted) odor in the nose in the absence of any external odor.

One type of olfactory hallucination was first reported to occur preceding an epileptic type of seizure and was called an "aura" or "breeze". This odor usually precedes the seizure by seconds, minutes or even hours but the hallucination usually lasts only seconds to minutes. This type of olfactory hallucination has been reported to smell rancid, fecal, burned, smoky, chemical or exceedingly sweet. This type of olfactory hallucination preceding a seizure is not uncommon but it is less common than a visual or auditory hallucination preceding a seizure. The seizure following the "aura" or hallucination is usually of a typical type with loss of consciousness and bodily shaking. This type of hallucination is usually transient. Following this seizure there is usually a period of recovery called a "postictal state" during which time the patient recovers from the effect of the seizure [1].

A second type of olfactory hallucination was reported to occur associated with a brain tumor or a specific brain lesion and may or may not be associated with a subsequent epileptic-type seizure. This type of olfactory hallucination is uncommon. It may last seconds to minutes and it is usually of the same character as is the olfactory hallucination preceding an epileptic seizure. This type of hallucination is also transient [2].

We have discovered a third type of olfactory hallucination with similar characteristics to those noted above. Just as with the preceding two types of olfactory hallucinations it can last seconds to minutes and its character is similar to the other hallucinations - rancid, fecal, burned, smoky, chemical or exceedingly sweet. However, it occurs independent of any subsequent seizure activity and independent of any brain tumor or brain lesion. Indeed, olfactory hallucinations independent of seizures or brain tumors are very common symptoms occurring in patients who have experienced a loss of smell. About $60 \%$ of patients who lose their smell acuity develop this type of olfactory hallucination usually after the acuity loss has been recognized. Since there may be as many as 21 million people in the United States who exhibit loss of smell this type of olfactory hallucination is quite common. This type of hallucination is usually persistent unlike the other two types of hallucinations [3].

Most patients with this third type of olfactory hallucination go to their local physician who will commonly consider this symptom related to the previous two types of medical problems - epilepsy or brain tumor. Clinical evaluations performed by most physicians who see patients with this third type of olfactory hallucination (e.g., brain MRI, neurological examination, tests of blood or urine) do not reveal its cause since most patients exhibit this symptom related to smell loss, not epilepsy or brain tumor, and most physicians are unaware of this smell relationship. Most physicians treat this hallucination based upon their knowledge of epilepsy; this treatment is unsuccessful since the cause of this symptom is usually loss of smell [4].

Since most physicians including neurologists are unfamiliar with this type of problem most patients are frustrated since their olfactory hallucination persists without adequate evaluation or treatment.

We have used repetitive transcranial magnetic stimulation (rTMS) to treat these patients with significant success in inhibiting these hallucinations among patients with this third type of olfactory hallucination [5].

\section{References}

1. Henkin RI, Potolicchio SJ, Levy LM (2013) Olfactory hallucinations without clinical motor activity: a comparison of unirhinal with birhinal phantosmia. Brain Sci 3: 1483-1553.

2. Henkin RI (2015) Taste and smell dysfunction in the United States. J Oto Rhino 4: 3.

3. Henkin RI, Levy LM, Fordyce A (2013) Taste and smell function in chronic disease: A review of clinical and biochemical evaluation of taste and smell dysfunction in over 5000 patients at The Taste and Smell Clinic in Washington, DC. Am J Otolaryngol 34: 477-489.

4. Henkin RI (1987) Taste and smell disorders. In: Adelman G, Ed. Encyclopedia of Neuroscience. Boston: Birkhauser.

5. Henkin RI, Potolicchio SJ, Levy LM (2011) Improvement in smell and taste dysfunction after repetitive transcranial magnetic stimulation. Am J Otolaryngo 32: $38-46$
*Corresponding author: Robert I Henkin, Director, The Taste and Smell Clinic, Washington D.C., USA, Tel: 202-364-4180; E-mail: doc@tasteandsmell.com

Received November 26, 2015; Accepted November 27, 2015; Published December 07, 2015

Citation: Henkin RI (2015) What is an Olfactory Hallucination?. J Epilepsy 1: e104 doi:10.4172/2472-0895.1000e104

Copyright: @ 2015 Henkin RI. This is an open-access article distributed unde the terms of the Creative Commons Attribution License, which permits unrestricted use, distribution, and reproduction in any medium, provided the original author and source are credited. 